\title{
MANIFOLDS OF FUNCTIONS DEFINED BY SYSTEMS OF ALGEBRAIC DIFFERENTIAL EQUATIONS*
}

BY

\author{
J. F. RITT
}

This paper is concerned with the development of a theory of systems of algebraic ordinary differential equations, analogous to the theory of algebraic manifolds.

We deal with any finite or infinite system of algebraic differential equations in the independent variable $x$ and the dependent variables $y_{1} \ldots$ $y_{n}$. We write each equation in the form

$$
F\left(x ; y_{1}, \cdots, y_{n}\right)=0 \text {, }
$$

where $F$ is a polynomial in the $y$ 's and any number of their derivatives. The coefficients in $F$ will be supposed to be functions of $x$ meromorphic in some given open region $\mathfrak{A}$, and belonging to a given field $\mathcal{F}$ of such functions. By a field, we understand a set of functions of $x$, not all zero, such that, given any function of the set, its derivative is also in the set, and such that, given any two functions, $f$ and $g$, of the set,

$$
f+g, f-g, f g, \frac{f}{g} \quad(\text { when } g \neq 0)
$$

are all in the set. $\dagger$

An expression like $F$, above, will be called a form. With respect to every form introduced into our work, we shall assume, unless the contrary is stated, that its coefficients belong to $\mathcal{F}$.

By a solution of a system of forms, we shall mean any set of functions, $y_{1}, \cdots, y_{n}$, analytic in some area contained in $\mathfrak{A}$, which cause all of the forms to vanish. $\ddagger$ The totality of solutions of a system of forms will be called the content of the system. The content of any system will be called a manifold. If $\Sigma$ and $\Sigma^{\prime}$ are two systems of forms such that every solution of $\Sigma$ is a solution of $\Sigma^{\prime}$, then $\Sigma^{\prime}$ will be said to hold $\Sigma$.

\footnotetext{
* Presented to the Society, April 18, 1930; received by the editors May 9, 1930.

$\dagger$ We do not assume, as Picard does in his construction of the Galois theory of linear differential equations, and as Landau does in his work on the factorization of linear differential forms, that $\mathcal{F}$ contains all constants. See Picard, Traité d'Analyse, 2d edition, vol. 3, p. 562.

$\ddagger$ An alternative definition of a solution would be any set of convergent series of powers of $x-a$, where $a$ is any point of $\mathfrak{Y}$, which cause the forms to vanish.
} 
A system $\Sigma$ of forms will be called irreducible if, $G$ and $H$ being any two forms such that $G H$ holds $\Sigma$, either $G$ holds $\Sigma$ or $H$ holds $\Sigma$. A system which is not irreducible will be called reducible. The system of equations obtained by equating the forms of a system to zero, and also the manifold which is the content of the system of forms, will be called reducible or irreducible according as the system of forms is reducible or irreducible.*

We can now state the chief result of the first part of our paper. Every manifold is composed of a finite number of irreducible manifolds. That is, given any system of forms, $\Sigma$, there exist a finite number of irreducible systems, $\Sigma_{1}, \cdots, \Sigma_{s}$, such that $\Sigma$ holds every $\Sigma_{i}$, and that every solution of $\Sigma$ is a solution of some $\Sigma_{i}$. The decomposition into irreducible manifolds is essentially unique.

Let us consider an example. The equation

$$
y^{\prime 2}-4 y=0,
$$

which has the general solution $y=(x-a)^{2}$ and a singular solution $y=0$, is a reducible system in the field of all constants. For neither $y^{\prime \prime}-2$ nor $y^{\prime}$ vanishes for all solutions of the equation, while $\left(y^{\prime \prime}-2\right) y^{\prime}$ does. The equation is equivalent to the two irreducible systems

$$
y^{\prime 2}-4 y=0, \quad y^{\prime \prime}-2=0,
$$

and

$$
y^{\prime 2}-4 y=0, \quad y^{\prime}=0 .
$$

The decomposition theorem follows from a lemma which bears a certain analogy to Hilbert's theorem on the existence of a finite basis for any infinite system of polynomials. We prove that if $\Sigma$ is an infinite set of forms then $\Sigma$ has a finite subset whose content is identical with that of $\Sigma$. $\dagger$

In the second part of our paper, we investigate the structure of an irreducible manifold. We obtain, for every irreducible system, a differential equation which we call the resolvent of the system. Finding all non-singular

* The property that we have used in defining irreducible manifold is, of course, analogous to a characteristic property of irreducible algebraic manifolds. Of the different treatments of algebraic manifolds, that of van der Waerden, loc. cit., seems to be the only one that uses this property as a defining property. By the method of the present paper, the theorem that every algebraic manifold consists of a finite number of irreducible manifolds can be proved in a manner even simpler than that of van der Waerden, without using Lasker's theorem.

$\dagger$ This result is very different in nature from that of Tresse for systems of partial differential equations. See Tresse, Acta Mathematica, vol. 18 (1894), p. 4. Also, Drach, Annales de l'Ecole Normale, vol. 34 (1898), p. 292. In solving his system algebraically for certain derivatives, Tresse has necessarily to confine himself to a portion of the content of his system. The chief feature of the present investigation is its completeness from the algebraic standpoint. 
solutions of the resolvent is equivalent to determining the content of the irreducible system.

To see what is contained in the idea of the resolvent, let us consider a differential equation $\alpha=0$, where $\alpha$ is a form in the $q+1$ indeterminates $w$; $u_{1}, \cdots, u_{q}$, irreducible, as a polynomial in $\mathfrak{F}$ in the indeterminates and their derivatives.

Let $\alpha$ be of $\operatorname{arder} r$ in $w$. Let $F=\partial \alpha / \partial w_{r}$, where $w_{r}$ is the $r$ th derivative of $w$. Let $H$ be the coefficient of the highest power of $w_{r}$ in $\alpha$. We call a solution of $\alpha=0$, for which neither $F$ nor $H$ vanishes, a regular solution.

We prove that the totality of forms which vanish for all regular solutions of $\alpha=0$ is an irreducible system. The content of this system is one of the irreducible manifolds in the content of $\alpha$. We call this irreducible manifold the general solution of $\alpha$.

Now, suppose that we have $p$ rational combinations of $w ; u_{1}, \cdots, u_{q}$ and their derivatives, with coefficients in $F$,

$$
y_{i}=R_{i}\left(w ; u_{1}, \cdots, u_{q}\right) \quad(i=1, \cdots, p),
$$

no denominator vanishing for all regular solutions of $\alpha=0$.

We prove that there exist forms in $u_{1}, \cdots, u_{q} ; y_{1}, \cdots, y_{p}$, which vanish for all $u^{\prime}$ 's and $y$ 's in (1), it being understood that $w ; u_{1}, \cdots, u_{q}$ belongs to the general solution of $\alpha$. The totality of these forms in the $u$ 's and $y$ 's constitutes an irreducible system.

Conversely, let us consider any irreducible system in $y_{1}, \cdots, y_{n}$. A certain number, $q$, of the $y$ 's are found to play the rôle of arbitrary functions in the content of the system. We call these $u_{1}, \cdots, u_{q}$, and designate the remaining $y$ 's by $y_{1}, \cdots, y_{p}(p+q=n)$.

We show that, if $F$ does not consist purely of constants, it is possibia to form a rational combination $w$ of the $u$ 's, $y$ 's and their derivatives, in such a way that $y_{1}, \cdots, y_{p}$ become rational combinations of $w ; u_{1}, \cdots, u_{q}$ and their derivatives. The new indeterminate, $w$, satisfies a differential equation

$$
\alpha\left(u_{1}, \cdots, u_{q} ; w\right)=0,
$$

with $\alpha$ irreducible as a polynomial in $\mathfrak{F}$. This equation is a resolvent of the irreducible system.

The introduction of the resolvent creates a perfect analogy between the notion of the content of a system of algebraic differential equations and the notion of algebraic function of several variables.

The resolvent can be used to advantage in the study of such questions as the influence on the reducibility of a system of the adjunction of new functions to $\mathcal{F}$. 
Certain features of our proofs indicate that a theory of ideals of differential forms underlies the manifold theory. We are at present investigating this question.*

One will notice that we do not furnish a method for resolving a system into irreducible systems, or a method for constructing the resolvent. These questions, also, we expect to treat in further communications.

Our work has, apparently, nothing in common with the researches of Riquier and others on the degree of generality of the solution of a system of partial differential equations. $f$ We reserve for later papers the extension of our results to partial differential equations. $\ddagger$

The irreducible differential equations of Koenigsberger, $\S$ and Drach's irreducible systems of partial differential equations, $\mathbb{T}$ are irreducible in the sense explained above. The definitions of Koenigsberger and of Drach, which demand much more for irreducibility than does ours, are the starting points of group-theoretic investigations, which parallel the Galois theory.॥ Our definition leads, as we have seen, in a different direction.

This paper has a degree of contact with the work on field theory and elimination theory of the modern school of German algebraists. We would mention particularly the writings of Steinitz, Emmy Noether, Schmeidler and van der Waerden.**

\section{PART I. RESOLUTION OF A SYSTEM INTO IRREDUCIBLE SYSTEMS}

\section{Classification of FORMS}

1. Derivatives of functions $y_{i}$ will be indicated by means of a second subscript. Thus

$$
y_{i j}=\left(d^{j} / d x^{j}\right) y_{i}
$$

We write, frequently, $y_{i}=y_{i 0}$.

By the $j$ th derivative of a form $F$, we mean the form obtained by differentiating $F j$ times with respect to $x$, regarding $y_{1}, \cdots, y_{n}$ as functions of $x$.

By the order of $F$ with respect to $y_{i}$, if $F$ involves $y_{i}$ or some of its deriva-

* In this connection we have recently proved that if $G$ holds the system $F_{1}, \cdots, F_{p}$, then some power of $G$ is a linear combination of the $F$ 's and their derivatives, with forms for coefficients. This is analogous to a theorem of Hilbert for polynomials. (Note added in proof, August 4,1930.)

† See Janet, Systèmes d'Equations aux Dérivées Partielles, Paris, 1929.

¥ We have extended the theorem on the equivalence of a system to a finite number of irreducible systems to partial differential equations. (Note added in proof, August 4, 1930.)

§ Lehrbuch der Differentialgleichungen, Leipzig, 1889.

T Loc. cit., p. 295.

|| In connection with Koenigsberger's definition, we have in mind the Picard-Vessiot theory.

** For references, see van der Waerden, Mathematische Annalen, vol. 97 (1927), p. 196. 
tives effectively, we shall mean the greatest $j$ such that $y_{i j}$ is present in a term of $F$ with a coefficient distinct from zero. If $F$ does not contain $y_{i}$, the order of $F$ with respect to $y_{i}$ will be taken as zero.

By the class of a form which effectively involves some of the $y$ 's, we shall mean the greatest $p$ such that some $y_{p j}$ is present in $F$. If $F$ is simply a function of $x, F$ will be said to be of class 0 .

Let $F_{1}$ and $F_{2}$ be two forms. If $F_{2}$ is of higher order than $F_{1}$ in some $y_{p}$, $F_{2}$ will be said to be of higher rank than $F_{1}$ in $y_{p}$. If $F_{1}$ and $F_{2}$ are of the same order, say $q$, in $y_{p}$, and if $F_{2}$ is of greater degree than $F_{1}$ in $y_{p q}{ }^{*}$ then, again, $F_{2}$ will be said to be of higher rank than $F_{1}$ in $y_{p}$. Two forms for which no difference in rank is established by the foregoing criteria, will be said to be of the same rank in $y_{p}$.

If $F_{2}$ is of higher class than $F_{1}, F_{2}$ will be said to be of higher rank than $F_{1}$. If $F_{2}$ and $F_{1}$ are of the same class $p>0$, and if $F_{2}$ is of higher rank than $F_{1}$ in $y_{p}$, then, again, $F_{2}$ will be said to be of higher rank than $F$. Two forms for which no difference in rank is created by the preceding, will be said to be of the same rank. $\dagger$

\section{COMPLETENESS OF INFinite Systems}

2. In $\$ \S 2-11$, we prove the following lemma:

Lемма. Every infinite set of forms in $y_{1}, \cdots, y_{n}$ has a finite subset whose content is identical with that of the infinite set.

An infinite system of forms whose content is identical with that of one of its finite subsets will be called complete. $\ddagger$ Systems which are not complete will be called incomplete. In what follows, we assume the existence of incomplete systems, and force a contradiction.

3. We prove the following lemma:

Lемма. Let $\Sigma$ be an incomplete system. Let a form $F$, not in $\Sigma$, and a subset $\Sigma^{\prime}$ of $\Sigma$ exist, such that the system $\Lambda$, composed of the forms of $\Sigma$ not in $\Sigma^{\prime}$ and of the products of the forms of $\Sigma^{\prime}$ by $F$, is complete. Then the system $\Sigma+F$, obtained by adjoining $F$ to $\Sigma$, is incomplete.

Suppose that $\Sigma+F$ is complete. Let

$$
F ; \quad G_{1}, \cdots, G_{p} ; H_{1}, \cdots, H_{q},
$$

where the $H$ 's, but not the $G$ 's, belong to $\Sigma^{\prime}$, be a subset of $\Sigma+F$ whose

* Considered as a polynomial in $y_{p q}$. If a form is identically zero (hence of order 0 in every $y_{p}$ ), it will be considered of degree 0 in every $y_{p 0}$. This leads to no difficulties.

$\dagger$ Thus all forms of class 0 are of the same rank.

$\ddagger$ If some finite subset has no solutions, the infinite set will be considered complete. 
content is identical with that of $\Sigma+F$. We evidently may, and we shall, assume that the content of

$$
G_{1}, \cdots, G_{p} ; F H_{1}, \cdots, F H_{q}
$$

is that of $\Lambda$. Now, let $K$ be a form of $\Sigma$ which does not hold

$$
G_{1}, \cdots, G_{p} ; H_{1}, \cdots, H_{q} .
$$

As $F K$ holds (3), and as (3) holds (4), certain solutions of (4) which are not solutions of $K$ must be solutions of $F$. Thus $K$ does not hold (2). This proves the lemma.

4. By a first form of a system of forms, not all zero, we shall mean a form of the system, not zero, whose rank is not greater than that of any other nonzero form of the system.

From among all incomplete systems in $y_{1}, \cdots, y_{n}$, we select one whose first forms have a rank not greater than the rank of the first forms of any other incomplete system. Let $\Sigma_{1}$ be such an incomplete system, and let $\alpha_{1}$ be one of its first forms.

Let $\alpha_{1}$ be of class $p_{1}$. Then $p_{1}>0$, else $\alpha_{1}$ would have no solutions and $\Sigma_{1}$ would be complete.

5. Let a system $\Sigma$ contain a form $\alpha$ of class $p>0$. We call $\Sigma$ reduced with respect to $\alpha$ if every form of $\Sigma$, distinct from $\alpha$, is of lower rank than $\alpha$ in $y_{p}$.

6. We prove the following lemma:

LеммA. Given any incomplete system $\Sigma$ which contains the $\alpha_{1}$ of $\$ 4$, there exists an incomplete system which has $\alpha_{1}$ for first form, is reduced with respect to $\alpha_{1}$, and contains every form of $\Sigma$ which is of lower rank than $\alpha_{1}$ in $y_{p_{1}}$.

Let $\alpha_{1}$ be of order $r$ in $y_{p_{1}}$. The $q$ th derivative of $\alpha_{1}$ will be of order $r+q$ in $y_{p_{1}}$ and will be linear in $y_{p_{1}, r+q}$, with $\partial \alpha_{1} / \partial y_{p_{1}, r}$ for coefficient of $y_{p_{1}, r+q}$. Let $F=\partial \alpha_{1} / \partial y_{p_{1}, r}$. Then $F$ is of lower rank than $\alpha_{1}$.

Now, $G$ being any form of $\Sigma$ of order higher than $r$ in $y_{p_{1}}$, it is possible, using the algorithm of division, to find a non-negative integer $m$,depending on $G$, such that, when we subtract from $F^{m} G$ a suitable linear combination of the derivatives of $\alpha_{1}$, with forms in $y_{1}, \cdots, y_{n}$ for coefficients, the remainder, call it $B$, is of order not greater than $r$ in $y_{p_{1}}$. Let such a $B$ be found for every $G$.

Let $\Omega$ be the system composed of all $B$ 's and of all forms of $\Sigma$ whose order in $y_{p_{1}}$ does not exceed $r$. We are going to show that $\Omega$ is incomplete.

Let $\Lambda$ be the system composed of the forms $F^{m} G$ and the forms of $\Sigma$ whose order in $y_{p_{1}}$ does not exceed $r$. 
Every $F^{m} G$ holds the system of two forms composed of its $B$ and $\alpha_{1}$. Every $B$ holds the system formed of its $F^{m} G$ and $\alpha_{1}$. Thus, if $\Omega$ were complete, $\Lambda$ would be complete. Now, if $m \geqq 1, F^{m} G$ and $F G$ hold each other. Thus, if $\Lambda$ were complete, the system obtained from $\Sigma$ by multiplying some subset of $\Sigma$ by $F$ would be complete. Then, by $\$ 3, \Sigma+F$ would be incomplete. This is impossible, because $F$ is of lower rank than $\alpha_{1}$ and is not identically zero. Thus $\Omega$ is incomplete.

Let $H$ be the coefficient of the highest power of $y_{p_{1}, r}$ in $\alpha_{1}$. Then $H$ is of lower rank than $\alpha_{1}$.

Let $K$ be any form of $\Omega$ which is not zero and which is not of lower degree than $\alpha_{1}$ in $y_{p_{1}, r}$. An integer $m \geqq 0$ exists such that

$$
H^{m} K=C \alpha_{1}+D,
$$

where $C$ and $D$ are forms in $y_{1}, \cdots, y_{n}$ and where $D$ is either zero or of lower degree than $\alpha_{1}$ in $y_{p_{1}, r}$. We see, as above, that the system composed of $\alpha_{1}$, the forms of $\Sigma$ which are of lower rank than $\alpha_{1}$ in $y_{p_{1}}$, and the $D$ 's, is incomplete. Furthermore, this system is reduced with respect to $\alpha_{1}$. The lemma is proved.

7. Consider any $B$ of $\Omega$, and the $G$ from which it is obtained.

We say that, if $q>p_{1}$, then $B$ is not of higher rank than $G$ with respect to $y_{q}$. Let

For instance, let $\beta$ be a derivative of $\alpha_{1}$, of the same order as $G$ in $y_{p_{1}}$.

$$
F^{m} G=A \beta+G_{1},
$$

where $G_{1}$ is of lower order than $G$ in $y_{p_{1}}$. Surely $G_{1}$ is not of higher order than $G$ in $y_{q}$. Suppose that $G$ and $G_{1}$ are of the same order, $h$, in $y_{q}$. If $G_{1}$ involved $y_{q h}$ in a higher power than $G$ does, then $A$ would contain the higher power of $y_{q h}$, so that $A \beta$ would contain the higher power of $y_{q h}$ multiplied by the derivative of highest order of $y_{p_{1}}$ in $\beta$. There would thus be terms in $A \beta$ which would not be balanced by the terms of $F^{m} G$ and $G_{1}$.

Similarly, consider any $D$ of the final system of $\$ 6$, and the $K$ which corresponds to it. We see that, if $q>p_{1}, D$ will not be of higher rank than $K$ in $y_{q}$.

The observations of this section will be of great utility in $\$ 9$.

8. Consider any incomplete system which has $\alpha_{1}$ for first form and is reduced with respect to $\alpha_{1}$. In such a system, there cannot be a non-zero form which is distinct from $\alpha_{1}$ and of class not exceeding $p_{1}$, for such a form would have to be of lower rank than $\alpha_{1}$.

It follows that $p_{1}<n$. 
Of the non-zero forms in the above system which are distinct from $\alpha_{1}$, there are some of lowest rank. Such forms, we call second forms of the system.

From among all incomplete systems which have $\alpha_{1}$ for first form, and are reduced with respect to $\alpha_{1}$, we choose one, $\Sigma_{2}$, whose second forms are of as low a rank as is possible. Let $\alpha_{2}$, of class $p_{2}>p_{1}$, be a second form of $\Sigma_{2}$.

9. We prove the following lemma:

Lemma. Given any incomplete system $\Sigma$ which contains $\alpha_{1}$ and $\alpha_{2}$, there exists an incomplete system containing $\alpha_{1}$ and $\alpha_{2}$, which is reduced with respect to $\alpha_{1}$ and $\alpha_{2}$, and which contains all forms of $\Sigma$ which are of lower rank than $\alpha_{2}$ in $y_{p_{2}}$ and of lower rank than $\alpha_{1}$ in $y_{p_{1}}$.

We note that the system whose existence is to be proved, being reduced with respect to $\alpha_{1}$, has $\alpha_{2}$ as a second form.

Let $\alpha_{2}$ be of order $r$ in $y_{p_{2}}$. Let $G$ be any form of order higher than $r$ in $y_{p_{2}}$. Let $F=\partial \alpha_{2} / \partial y_{p_{2}, r}$. Then there is an $m \geqq 0$ such that when a suitable linear combination of the derivatives of $\alpha_{2}$ is subtracted from $F^{m} G$, the remainder, $B$, has an order in $y_{p_{2}}$ not greater than that of $\alpha_{2}$. The system $\Omega$ composed of the $B$ 's and the forms of $\Sigma$ not of higher order than $r$ in $y_{p_{2}}$ must be incomplete. If not, $\Sigma+F$ would be incomplete. Now $F$, like $\alpha_{2}$, is of lower rank than $\alpha_{1}$ in $y_{p_{1}}$. By $\S 6$, there would be an incomplete system with $\alpha_{1}$ for first form, reduced with respect to $\alpha_{1}$ and containing $F$. This is impossible if the class of $F$ does not exceed $p_{1}$, for then $F$ must be of lower rank than $\alpha_{1}$. It is impossible if the class of $F$ exceeds $p_{1}$, since $F$ is of lower rank than $\alpha_{2}$. Thus $\Omega$ must be incomplete.

Again, if $H$ is the coefficient of the highest power of $y_{p_{2}, r}$ in $\alpha_{2}$, we have, for any form $K$ of $\Omega$, distinct from zero and of degree in $y_{p_{2}, r}$ not less than that of $\alpha_{2}$,

$$
H^{m} K=C \alpha_{2}+D
$$

with $D$ either zero or of lower degree than $\alpha_{2}$ in $y_{p_{2}, r}$.

We shall show that the $D$ 's, $\alpha_{2}$ and the forms of $\Omega$ which are of lower rank than $\alpha_{2}$ in $y_{p_{2}}\left(\alpha_{1}\right.$ is among them) constitute an incomplete system $\boldsymbol{\Xi}$.

For, if $\Xi$ were complete, $\Omega+H$ would be incomplete. By $\S 6$, there would exist a system containing $\alpha_{1}$, reduced with respect to $\alpha_{1}$, and containing $H$. As was seen above, this is impossible.

Proceeding now with $\alpha_{1}$ as in $\S 6$, and operating on the forms of $\Xi$ of rank in $y_{p}$ not less than that of $\alpha_{1}$, we obtain an incomplete system containing $\alpha_{1}$, $\alpha_{2}$ and all forms of $\Sigma$ which are of lower rank than $\alpha_{2}$ in $y_{p_{2}}$ and of lower rank than $\alpha_{1}$ in $y_{p_{1}}$, the system being reduced with respect to $\alpha_{1}$. Now this 
system is also reduced with respect to $\alpha_{2}$, for, as was seen in $\S 7$, when we operate on a form of $\Xi$ with $\alpha_{1}$, the new form obtained has a rank with respect to $y_{p_{2}}$ not greater than that of the original form. This proves the lemma.

10. Evidently an incomplete system containing $\alpha_{1}$ and $\alpha_{2}$, and reduced with respect to $\alpha_{1}$ and $\alpha_{2}$, contains no non-zero form other than $\alpha_{1}$ and $\alpha_{2}$ whose class does not exceed $p_{2}$.

We conclude that $p_{2}<n$.

In the incomplete systems of the type just described, we call those forms whose class exceeds $p_{2}$, and whose rank is as low as it can be, with this condition, third forms.

We select a system $\Sigma_{3}$ with a third form $\alpha_{3}$ of as low a rank as is possible. We operate as above, obtaining an incomplete system which contains $\alpha_{1}, \alpha_{2}$, $\alpha_{3}$ and is reduced with respect to $\alpha_{1}, \alpha_{2}, \alpha_{3}$. It follows, if $\alpha_{3}$ is of class $p_{3}$, that $p_{3}<n$.

11. Continuing in this fashion, we find that there exists an infinite sequence of integers

$$
p_{1}<p_{2}<p_{3}<\cdots,
$$

all less than $n$. This absurdity proves the truth of the fundamental lemma stated in $\$ 2$.

\section{NoN-existence of a Hilbert theorem}

12. It might be conjectured that in every system $\Sigma$ there is a finite system of forms such that every form of $\Sigma$ is a linear combination of the forms of the finite system, and their derivatives, with forms for coefficients. We shall show that this is not so.

We consider forms in a single dependent variable, $y$, and represent the $n$th derivative of $y$ by $y_{n}$.

Consider the system

$$
y_{1} y_{2}, y_{2} y_{3}, \cdots, y_{n} y_{n+1}, \cdots .
$$

We shall show that no form of this system with $n>1$ is linearly expressible in terms of the forms which precede it, and their derivatives.

We notice that all of the forms, and all of their derivatives, are homogeneous polynomials of the second degree in the $y$ 's. Also if the weight of $y_{i} y_{j}$ is defined as $i+j$, the $p$ th derivative of $y_{i} y_{j}$ will be isobaric, with its terms of weight $i+j+p$.

Now if

$$
y_{n} y_{n+1}=A_{1} y_{1} y_{2}+\cdots+A_{n-1} y_{n-1} y_{n}+B_{1} \frac{d}{d x}\left(y_{1} y_{2}\right)+\cdots,
$$


with the $A$ 's, $B$ 's, etc., forms, the terms not independent of the $y$ 's in the $A$ 's, etc., may be cast out, for they produce terms of degree greater than 2 .

Again, considering the weights of the various forms, we find that

$$
y_{n} y_{n+1}=C_{1} \frac{d^{2 n-2}}{d x^{2 n-2}}\left(y_{1} y_{2}\right)+\cdots+C_{n-1} \frac{d^{2}}{d x^{2}}\left(y_{n-1} y_{n}\right)
$$

with $C$ 's independent of the $y$ 's. Now the $(2 n-2)$ d derivative of $y_{1} y_{2}$ contains a term $y_{1} y_{2 n}$, and none of the other derivatives in (5) yields such a term. We conclude that $C_{1}=0$. Continuing, we find every $C$ to be zero. This proves our statement.

\section{IRREDUCIBLE SYSTEMS}

13. We prove the following fundamental theorem:

THEOREM. Given any system $\Sigma$ of forms in $y_{1}, \cdots, y_{n}$, there exist a finite number of irreducible systems, $\Sigma_{1}, \cdots, \Sigma_{s}$, such that $\Sigma$ holds every $\Sigma_{i}$, while every solution of $\Sigma i$ s a solution of some $\Sigma_{i}$.

Let the theorem be false for some system $\Sigma$. Then $\Sigma$ is reducible. Let $G_{1}$ and $G_{2}$ be two forms such that $G_{1} G_{2}$, but neither $G_{1}$ nor $G_{2}$, holds $\Sigma$. Now $\Sigma$ holds $\Sigma+G_{1}$, and $\Sigma+G_{2}$, and every solution of $\Sigma$, being a solution of $G_{1}$ or of $G_{2}$, is a solution of $\Sigma+G_{1}$ or of $\Sigma+G_{2}$.

Thus at least one of the systems $\Sigma+G_{1}$ or $\Sigma+G_{2}$ is reducible. If either of these systems is reducible, we call it a system of the first class. There must be a system of the first class which, when treated like $\Sigma$, yields either one or two reducible systems, obtained by adjoining two forms to $\Sigma$. The reducible systems obtained through two adjunctions, we call systems of the second class. Some of the systems of the second class, when treated like $\Sigma$, must yield reducible systems obtained from $\Sigma$ by three adjunctions. We call these systems of the third class. We proceed in this manner, forming systems of all classes.

There must be a system of the first class whose forms are contained in systems of all classes higher than the first. Let $\Sigma+H_{1}$, where $H_{1}$ is either $G_{1}$ or $G_{2}$, be such a system of the first class. One of the systems of the second class which contains the forms of $\Sigma+H_{1}$ must have its forms contained in systems of all classes higher than the second. Let $\Sigma+H_{1}+H_{2}$ be such a system. Let an $H_{p}$ be found in this way for every $p$. Then the system composed of

$$
\Sigma, H_{1}, H_{2}, \cdots, H_{p}, \cdots
$$

is incomplete. This proves our theorem. It will be noticed that the proof involves making an infinite number of selections. 


\section{UNIQUENESS OF RESOLUTION}

14. We suppose, suppressing certain of the irreducible systems $\Sigma_{i}$, if necessary, that no $\Sigma_{i}$ holds a $\Sigma_{j}$ with $j \neq i$.

It is then possible to prove that the systems $\Sigma_{i}$ are essentially unique; that is, if $\Omega_{1}, \cdots, \Omega_{i}$ is a second set of irreducible systems, none of which holds any other, each of which is held by $\Sigma$, and which are such that every solution of $\Sigma$ is a solution of some $\Omega_{i}$, then $s=t$, and every $\Omega_{i}$ holds, and is held by, some $\Sigma_{i}$.

We shall show that there is some $\Omega_{i}$ which holds $\Sigma_{1}$. If there were not, then each $\Omega_{i}$ would have a form which would not hold $\Sigma_{1}$. Such forms being selected, their product would hold each $\Omega_{i}$, consequently $\Sigma$, thus $\Sigma_{1}$. This is impossible if $\Sigma_{1}$ is irreducible and none of the forms holds $\Sigma_{1}$.

Then let $\Omega_{1}$ hold $\Sigma_{1}$. Now $\Omega_{1}$, similarly, must be held by some $\Sigma_{i}$, which must be $\Sigma_{1}$, since no $\Sigma_{i}$ with $i \neq 1$ holds $\Sigma_{1}$. Thus $\Omega_{1}$ and $\Sigma_{1}$ hold each other. The uniqueness is proved.

\section{PART II. STRUCTURE OF AN IRREDUCIBLE MANIFOLD}

\section{General SOlution of a SINGLE EQUATION}

15. We are going to study the content of a single form, $\alpha$, of class $n>0$. We assume that $\alpha$ is an irreducible form, that is, that $\alpha$ cannot be expressed as the product of two forms, each of class greater than 0 , and each with coefficients in $F$.

It is our object to make precise the notion of the general solution of $\alpha=0$.

We write $y_{n}=y$, and, if $n>1$, we write $q=n-1, y_{i}=u_{i}, i=1, \cdots, n-1$.

Our definition of the general solution will appear, at first, to depend on the order in which the dependent variables in $\alpha$ are taken; at least, on the manner in which $y$ is selected from among the dependent variables. But it will turn out, finally, that the definition is actually independent of such order.

Let $\alpha$ be of order $r$ in $y$. Let $F=\partial \alpha / \partial y_{r}$, where $y_{r}$ is the $r$ th derivative of $y$, and let $H$ be the coefficient of the highest power of $y_{r}$ in $\alpha$. A solution of $\alpha$ which is not a solution of $F$ or of $H$ will be called a regular solution of $\alpha$.

Let $A$ and $B$ be forms in $u_{1}, \cdots, u_{q} ; y$, which are such that every regular solution of $\alpha$ is a solution of $A B$.

We shall prove that either every regular solution of $\alpha$ is a solution of $A$ or every regular solution is a solution of $B$.

From Part I (\$6) we know that there exists a form $A_{1}$, of lower rank 
than $\alpha$, such that every regular solution of $\alpha$ which satisfies either of the equations $A=0$ or $A_{1}=0$, satisfies the other. For $B$, similarly, we find a form $B_{1}$, of lower rank than $\alpha$.

If, then, we can show that either $A_{1}$ or $B_{1}$ is zero identically, our result will be proved.

Suppose that neither $A_{1}$ nor $B_{1}$ is identically zero. Consider any set of numerical values of $x$ and of $u_{1}, \cdots, u_{q} ; y$ and their derivatives appearing in $\alpha, A_{1}, B_{1}$, for which $\alpha=0$. Let the value of $x$ be $a$. Suppose that neither $F$ nor $H$ vanishes for these numerical values.*

We construct functions $u_{1}, \cdots, u_{q}$ which have for themselves and for their derivatives, at $a$, the values indicated above. The existence theorem for differential equations assures us that $\alpha$ has a regular solution in which the $u$ 's, $y$ and their derivatives have the indicated values at $a . \dagger$ This means that the set of numerical values makes either $A_{1}$ or $B_{1}$ vanish. All in all, $A_{1} B_{1} F H$ vanishes for all numerical values for which $\alpha$ vanishes. This means, since $\alpha$ is an irreducible form, that $A_{1} B_{1} F H$ is the product of $\alpha$ by a form. This cannot be, since none of $A_{1}, B_{1}, F, H$ can be divisible by $\alpha$. This completes the proof.

16. It follows immediately, from $\$ 15$, that the system of all forms in $u_{1}, \cdots, u_{q} ; y$, which vanish for all regular solutions of $\alpha$, is an irreducible system. The irreducible manifold which is the content of this irreducible system will be called the general solution of $\alpha=0$ (or of $\alpha$ ).

We show that every solution of $\alpha$, for which $F$ does not vanish, belongs to the general solution.

Let $A$ be any form which vanishes for all regular solutions. As above, let $\alpha$ be of order $r$ in $y$. Let $A_{1}$ be a form, not of order greater than $r$ in $y$, which differs from some $F^{m} A$ by a linear combination of the derivatives of $\alpha$. Some $H^{*} A_{1}$ equals the product of $\alpha$ by a form, plus a form $A_{2}$ of lower rank than $\alpha$. As $A_{2}$ vanishes for all regular solutions of $\alpha, A_{2}$, by an argument used in $\$ 15$, must be identically zero. Thus $H^{s} A_{1}$ is divisible by $\alpha$. This means, since $\alpha$ is an irreducible form, and $H$ is not divisible by $\alpha$, that $A_{1}$ is divisible by $\alpha$. Thus $A_{1}$ holds $\alpha$. Hence $A$ vanishes for all solutions of $\alpha$ for which $F$ does not vanish. This proves our statement.

We shall prove that the general solution of $\alpha$ is not contained in any other irreducible manifold of solutions of $\alpha$.

Let $M$ be any irreducible manifold of solutions of $\alpha$ which contains the general solution. Those solutions in $M$ which are not in the general solution

* We are assuming that the coefficients in $\alpha, A_{1}, B_{1}, F, H$ are all analytic at $a$.

$\dagger$ That is, when the functions $u$ are constructed, we regard $\alpha=0$ as an equation in $y$. 
make $F$ vanish. Let $B$ be any form which vanishes for every solution in the general solution. Then $B F$ vanishes for every solution in $M$. Since $F$ does not vanish for every solution in $M, B$ must. Thus $M$ is identical with the general solution.

We shall prove that the definition of the general solution is independent of the order in which the indeterminates $y_{1}, \cdots, y_{n}$ are taken.

Let $M_{1}, \cdots, M_{s}$ be $s>1$ irreducible manifolds, none containing any other, which make up the content of $\alpha{ }^{*}$ Suppose that, when $y=y_{n}$, the general solution is $M_{1}$, whereas, when $y=y_{1}$, the general solution is $M_{8}$.

Let $F^{\prime}$ have, relative to $y_{1}$, the definition which $F$ has relative to $y_{n}$. Then every solution in $M_{1}$ must make $F^{\prime}$ vanish. For, let $B$ be a form which vanishes for every solution in $M_{s}$, but not for every solution in $M_{1}$. Then, since $B F^{\prime}$ vanishes for every solution of $\alpha, F^{\prime}$ must vanish for every solution of $M_{1}$.

This means that every set of numerical values of $x$, the $y$ 's and their derivatives, which makes $\alpha$ vanish, and which does not make $F$ vanish, makes $F^{\prime}$ vanish; that is, for numerical values for which $\alpha$ vanishes, $F F^{\prime}$ vanishes. For, according to the existence theorem for differential equations, a set of numerical values with $\alpha=0$ and $F \neq 0$ furnishes a solution in $M_{1}$.

Then, since $\alpha$ is an irreducible form, $F F^{\prime}$ must be divisible, as a polynomial in the $y$ 's and their derivatives, with coefficients in $\mathcal{F}$, by $\alpha$. This is impossible, for neither $F$ nor $F^{\prime}$ can be divisible by $\alpha$. Our statement is proved.

\section{Analytic CONSTitution of the General solution $\dagger$}

17. A solution $\bar{u}_{1}, \cdots, \bar{u}_{q} ; \bar{y}$ of $\alpha$, for which either $F$ or $H$ vanishes, will be said to be semi-regular if there exists a set of points, dense in the area, contained in $\mathfrak{A}$, in which the functions of the solution are analytic, such that, given any point $a$ of the set, any positive integer $m$, and any $\epsilon>0$, there exists a regular solution $u_{1}, \cdots, u_{q} ; y$ (analytic at $a$ ) such that

(6) $\left|u_{i j}(a)-\bar{u}_{i j}(a)\right|<\epsilon,\left|y_{j}(a)-\bar{y}_{j}(a)\right|<\epsilon(i=1, \cdots, q ; j=0, \cdots, m)$.

Here, $u_{i j}$ is the $j$ th derivative of $u_{i}$, and $y_{j}$ the $j$ th derivative of $y\left(u_{i 0}=u_{i}\right.$, $\left.y_{0}=y\right)$.

Any solution for which $H=0$, but for which $F$ does not vanish, is semiregular. This is an immediate consequence of the implicit function theorem (applied to $\alpha$ with respect to $y_{r}$ ) and of the theorem on the differentiability

\footnotetext{
* When $s=1$ we have our result immediately.

$\dagger$ The results of $\S \S 17,18$, and the analogous results of $\S 24$, have contact with the remainder of the paper only in $\$ 28$.
} 
of the solution of a differential equation with respect to the constants of integration.

Let $A$ be any form in $u_{1}, \cdots, u_{q} ; y$ with coefficients meromorphic in $\mathfrak{A}$. The coefficients in $A$ need not belong to $F$. Suppose that $A$ vanishes for every regular solution of $\alpha$. We shall prove that $A$ vanishes for every semi-regular solution of $\alpha$.

Consider any semi-regular solution $\bar{u}_{1}, \cdots, \bar{u}_{q} ; \bar{y}$, and the points $a$ described above. Since the points are dense in an area, we can choose a point $a$ at which the coefficients in $A$ are analytic. Let this be done. When $\bar{u}_{1}, \cdots, \bar{u}_{q} ; \bar{y}$ are substituted into $A, A$ becomes a function $\phi(x)$ of $x$, which is zero at $a$. This is because $A$ vanishes for all regular solutions, and because of the $m, \epsilon$ item in the definition of semi-regular solution. Again, $\phi^{\prime}(x)$ must be zero at $a$, because the form obtained differentiating $A$ with respect to $x$ vanishes for every regular solution. Similarly, every derivative of $\phi(x)$ is zero at $a$. This proves that $A$ vanishes for the semi-regular solution.

If we restrict ourselves to forms $A$ with coefficients in $\mathcal{F}$, we see that the semi-regular solutions of $\alpha$ belong to the general solution.

18. We are going to prove that the general solution of $\alpha$ is composed of the regular solutions and of the semi-regular solutions.*

We denote by $\alpha_{j}$ the $j$ th derivative of $\alpha$. If $\alpha$ is of order $r$ in $y$, then $\alpha_{i}$ is of order $r+j$ in $y$. It is linear in $y_{r+j}$, the coefficient of $y_{r+j}$ being $F$. Also the order of $\alpha_{j}$ in each $u$ effectively present in $\alpha$ exceeds the corresponding order of $\alpha$ by $j$.

We shall examine the system of equations

$$
\alpha=0, \alpha_{1}=0, \cdots, \alpha_{s}=0,
$$

where $s$ is any positive integer, considering the equations not as differential equations, but merely as algebraic equations among a set of indeterminates $u_{i j}, y_{j}$. That is, any set of functions $u_{i j}, y_{j}$, analytic in some area in $\mathfrak{A}$, and satisfying (7), will be considered as a solution of (7). We do not ask, for instance, that $y_{j}$ be the derivative of $y_{j-1}$.

We know from the theory of algebraic manifolds that the solutions of (7) form a finite number of irreducible manifolds. An irreducible manifold, here, is the totality of solutions of a set of algebraic equations in the $u_{i j}$ 's and $y_{j}$ 's appearing in (7) (coefficients in $F$ ) the set of equations being such that if $A B$ vanishes for all of its solutions, where $A$ and $B$ are polynomials

\footnotetext{
* W. Weltmann, Archiv der Mathematik und Physik, vol. 58(1876), p. 337, defined a solution of an equation of the first order as singular if it cannot be approximated by solutions distinct from itself.
} 
with coefficients in $\mathcal{F}$, then either $A$ vanishes for all solutions or $B$ does.* We may and shall assume that none of the irreducible manifolds contains any other.

One of these irreducible manifolds must contain the general solution of $\alpha$. That is, there is one irreducible manifold such that, $u_{10}, \cdots, u_{q 0} ; y_{0}$ being in the general solution of $\alpha$, the irreducible manifold contains a solution $u_{i j}, y_{j}$, with $u_{i j}$ the $j$ th derivative of $u_{i 0}$ and $y_{i}$ the $j$ th derivative of $y_{0}$.

Suppose that this is not so. Let $M_{1}, \cdots, M_{t}$ be the irreducible manifolds of which the solutions of (7) are composed, and let $A_{i}, i=1, \cdots, t$, be a form which vanishes for every solution in $M_{i}$, but not for every regular solution of $\alpha$. As the general solution of $\alpha$ is an irreducible manifold, there are regular solutions which do not make $A_{1} \cdots A_{t}$ vanish. This contradicts the fact that every solution of $\alpha$ gives a solution of (7).

We shall identify an irreducible manifold $M$ of the solutions of (7) which contains the general solution of $\alpha$.

We call any solution of (7) for which neither $F$ nor $H$ vanishes, a regular solution of (7).

The equations (7) define $y_{r}, \cdots, y_{r+s}$ in terms of $y, \cdots, y_{r-1}$ and the $u_{i j}$ 's. That is, if we let $y, \cdots, y_{r-1}$ and the $u_{i j}$ 's be any functions, analytic in an area in $\mathscr{A}$, which do not render zero the resultant of $\alpha$ and $F$ with respect to $y_{r}, \dagger(7)$ determines $y_{r}, \cdots, y_{r+s}$, in succession, furnishing a regular solution.

It follows from the general theory of algebraic manifolds that there is only one irreducible manifold of solutions of (7) whose solutions do not all make $F$ vanish. This irreducible manifold, which contains the regular solutions of (7), is the manifold $M$ we have been seeking. Furthermore, in addition to the regular solutions of (7), $M$ contains those solutions of (7) which have the property that, in every area in which they are analytic, there is an area in which they can be approximated arbitrarily closely by a regular solution. $\ddagger$

Suppose then that $\bar{u}_{1}, \cdots, \bar{u}_{q} ; \bar{y}$ is a solution in the general solution of

* It should be emphasized that $A$ and $B$ involve only the indeterminates in (7), and not their derivatives.

$\dagger$ This resultant vanishes if either $F$ or $H$ vanishes.

$\ddagger$ It is known that, given a system of algebraic functions $z_{1}, \cdots, z_{t}$, of several variables, the values of $z_{1}, \cdots, z_{t}$ where they are analytic, together with all sets of $t$ numbers which can be approximated arbitrarily closely by such values, form an irreducible manifold in the field of all complex numbers. A similar result holds when the coefficients in the equations which determine the $z$ 's are not constants, but analytic functions, and when the values of the $z$ 's are analytic functions. 
$\alpha$. Consider any area $P$ in which the solution is analytic. Let an $m$ and an $\epsilon$ be assigned, as in the definition of semi-regular solution. Take $s>m$, and consider the corresponding system (7). Let a regular solution $\tilde{u}_{i j}, \tilde{y}_{j}$ of (7) be found, analytic in some area $P_{1}$ in $P$, such that, in $P_{1}$,

$$
\left|\tilde{u}_{i j}-\bar{u}_{i j}\right|<\epsilon,\left|\tilde{y}_{j}-\bar{y}_{j}\right|<\epsilon
$$

for all subscripts appearing in (7). ${ }^{*}$ We may and shall suppose that $F$ and $H$ are distinct from zero throughout $P_{1}$, for the solution $\tilde{u}_{i j}, \tilde{y}_{j}$. Let $a$ be any point of $P_{1}$. Let functions $u_{1}, \cdots, u_{q}$ be taken, analytic at $a$, so that $u_{i j}(a)=\tilde{u}_{i j}(a)$ for all subscript pairs appearing in (8). We notice that, for each $u_{i}, j$ assumes values at least as great as $m . \dagger$ Then, by (7), the differential equation $\alpha=0$ has a regular solution with $u_{1}, \cdots, u_{q}$ as just taken, and with $y_{j}(a)=\tilde{y}_{j}(a), j=0, \cdots, r+s$. Thus, for the given $m$ and $\epsilon$, any point in $P_{1}$ will serve as the point $a$ in the definition of semi-regular solution. Now, using $2 m$ and $\epsilon / 2$, we can find an area $P_{2}$, interior to $P_{1}$, any point $a$ of which can be used as above. Similarly, using $\epsilon / 4$ and $4 m$, we find an area $P_{3}$ in $P_{2}$, etc. There is a point $a$ which is interior to every $P_{i}$. Given any $\epsilon>0$, and any $m$, the differential equation $\alpha=0$ has a regular solution, analytic at $a$, for which (6) holds.

Thus, every solution of the general solution is either regular or semiregular.

\section{The BaSIC Equations}

19. We consider a system $\Sigma$ of forms in $y_{1}, \cdots, y_{n}$, not all zero. We assume that $\Sigma$ has solutions and that $\Sigma$ contains every form which holds $\Sigma$. For the rest, $\Sigma$ may be reducible or irreducible.

There may be some $y$, say $y_{i}$, such that no form of $\Sigma$ involves only $y_{i}$; that is, every form in which $y_{i}$ appears effectively also involves effectively some $y_{j}$ with $j \neq i$. If there exist such indeterminates $y_{i}$, let us pick one of them, arbitrarily, and call it $u_{1}$.

There may be a $y$, distinct from $u_{1}$, such that no form involves only $u_{1}$ and the new $y$. Let any such second $y$, if one or more exist, be denoted by $\boldsymbol{u}_{2}$.

Continuing in this way, we find a set, $u_{1}, \cdots, u_{q}(q<n)$, such that no form of $\Sigma$ involves any of the $u$ 's alone. Let the remaining indeterminates be represented now by $y_{1}, \cdots, y_{p}, p+q=n$. Then, given any $y_{i}$ among $y_{1}, \cdots, y_{p}$, there is a form in $\Sigma$ which involves only $y_{i}$ and the $u$ 's.

It will be seen, in $\$ 26$, that, when $\Sigma$ is irreducible, $q$ does not depend on the particular way in which the $u$ 's may be selected.

* If a $u_{i k}$ appears effectively in $\alpha$, every $u_{i j}$ with $j \leqq k$ is regarded as present in $\alpha$. Similarly for $y$.

$\dagger$ No difficulty arises here if a $u_{i}$ is not present effectively in $\alpha$. 
In what follows, we shall speak, generally, as if $u$ 's exist. It will be easy to see, in every case, what slight modifications of language are necessary when there are no $u$ 's.

Of all forms, not zero, in $\Sigma$, which involve no indeterminates other than $y_{1}$ and the $u$ 's, let $\alpha_{1}$ have a minimum rank in $y_{1}$.

There exist forms (not zero), involving only $y_{1}, y_{2}$ and the $u$ 's, which are of lower rank in $y_{1}$ than $\alpha_{1}$. For instance, any form involving only $y_{2}$ and the $u$ 's is of this type. Of all such forms, let $\alpha_{2}$ have a minimum rank in $y_{2}$.

Continuing in this way, we find a sequence of forms,

$$
\alpha_{1}, \alpha_{2}, \cdots, \alpha_{p}
$$

where

(I) $\quad \alpha_{i}$ involves only the $u$ 's and $y_{1}, \cdots, y_{i}$;

(II) $\alpha_{1}$ is of a minimum rank in $y_{1}$;

(III) for $i>1, \alpha_{i}$ is of lower rank in $y_{j}$ than $\alpha_{j}, j=1, \cdots, i-1$;

(IV) for $i>1, \alpha_{i}$ is not of greater rank in $y_{i}$ than any other form with the properties (I) and (III).

We shall call (9) a basic system.

If $\alpha_{i}$ is of order $r_{i}$ in $y_{i}$, we let $F_{i}=\partial \alpha_{i} / \partial y_{i, r_{i}}$. We designate the coefficient of the highest power of $y_{i, r_{i}}$ in $\alpha_{i}$ by $H_{i}$.

No $F_{i}$ can belong to $\Sigma$, for $F_{i}$ is of lower rank than $\alpha_{i}$ in $y_{i}$, of lower rank than $\alpha_{i-1}$ in $y_{i-1}$, etc. Similarly, no $H_{i}$ can belong to $\Sigma$.

A solution of the system (9) for which no $F_{i}$ or $H_{i}$ vanishes will be called a regular solution of (9).

We are going to show that every regular solution of (9) is a solution of $\Sigma$.

Consider any form $\beta$ of $\Sigma$ which involves only $y_{1}$ and the $u$ 's. There exist an $m$ and an $s$ such that, when a suitable linear combination of $\alpha_{1}$ and its derivatives is subtracted from $F_{1}^{m} H_{1}^{s} \beta$, the remainder, call it $\gamma$, is of lower rank than $\alpha_{1}$ in $y_{1}$. Then $\gamma$, which belongs to $\Sigma$, must be identically zero. Hence every solution of $\alpha_{1}$ for which $F_{1} H_{1}$ does not vanish is a solution of $\beta$.

Consider any form $\beta$, of $\Sigma$, which involves only $y_{1}, y_{2}$ and the $u$ 's. We find, as above, a $\gamma$, belonging to $\Sigma$, involving no $y_{i}$ with $i>2$ and of lower rank than $\alpha_{2}$ in $y_{2}$, such that every solution of $\alpha_{2}=0, \gamma=0$, for which $\mathrm{F}_{2} \mathrm{H}_{2}$ does not vanish is a solution of $\beta$. Furthermore, for $m$ and $s$ appropriate, $F_{1}^{m} H_{1}^{s} \gamma$ is a linear combination of $\alpha_{1}$ and its derivatives, plus a form $\delta$ of lower rank than $\alpha_{2}$ in $y_{2}$ and of lower rank than $\alpha_{1}$ in $y_{1}$. (See $\S 7$.) Then $\delta=0$. Thus every $u_{1}, \cdots, u_{q} ; y_{1}$ for which $\alpha_{1}=0$, and for which $F_{1} H_{1}$ does not vanish, makes $\gamma=0$ for any $y_{2}$. Thus a solution of $\alpha_{1}=0, \alpha_{2}=0$, for which none of $F_{1}, F_{2}, H_{1}, H_{2}$ vanishes is a solution of $\beta$. 
Continuing in this way, we see that every regular solution of (9) is a solution of $\Sigma$.

20. Suppose now that $\Sigma$ is irreducible. As no $F_{i}$ or $H_{i}$ holds $\Sigma$, the product of all $F$ 's and $H$ 's does not hold $\Sigma$.

It follows that (9) has regular solutions.

Furthermore, if a form vanishes for all regular solutions of (9), the form is in $\Sigma$. For, if $G$ is such a form,

$$
G F_{1} \cdots F_{p} H_{1} \cdots H_{p}
$$

holds $\Sigma$, so that $G$ holds $\Sigma$.

\section{THE RESOLVENT}

21. From now on, we shall understand, unless the contrary is stated, that $\mathcal{F}$ contains at least one function which is not a constant.

Let $\Sigma$ be reducible or irreducible, but not without solutions. We understand, as above, that $\Sigma$ contains every form which holds $\Sigma$.

We are going to show the existence, in $\mathcal{F}$; of functions

$$
\mu_{1}, \cdots, \mu_{p}
$$

and the existence of a form $G$, in the u's alone, such that, given two solutions of $\Sigma$ with the same $u$ 's,

$$
\begin{aligned}
& u_{1}, \cdots, u_{q} ; y_{1}{ }^{\prime}, \cdots, y_{p}{ }^{\prime}, \\
& u_{1}, \cdots, u_{q} ; y_{1}{ }^{\prime \prime}, \cdots, y_{p}{ }^{\prime \prime},
\end{aligned}
$$

for the $u^{\prime}$ 's of which $G$ does not vanish, and in which, for some $i, y_{i}^{\prime}$ is not identical with $y_{i}^{\prime \prime}$, then

$$
\mu_{1}\left(y_{1}^{\prime}-y_{1}^{\prime \prime}\right)+\cdots+\mu_{p}\left(y_{p}^{\prime}-y_{p}^{\prime \prime}\right)
$$

is not zero.*

We consider the system of forms obtained from $\Sigma$ by replacing each $y_{i}$ by a new indeterminate $z_{i}$. We take the system $\Omega$ composed of the forms of $\Sigma$, the forms in the $z$ 's just described, and also the form

$$
\lambda_{1}\left(y_{1}-z_{1}\right)+\cdots+\lambda_{p}\left(y_{p}-z_{p}\right)
$$

in which the $\lambda$ 's are indeterminates. That is, $\Omega$ involves $3 p+q$ indeterminates, namely, the $u$ 's, $y$ 's, $z$ 's, $\lambda$ 's.

Let $\Lambda$ be any irreducible system which $\Omega$ holds. We understand that $\Lambda$ contains every form which holds $\Lambda$.

Suppose that some one of the forms $y_{i}-z_{i}$ does not hold $\Lambda$. We shall

* Naturally, we assume that the two solutions have a common domain of analyticity. 
prove that $\Lambda$ contains a non-zero form which involves no indeterminates other than the $u$ 's and the $\lambda$ 's.

Since $\Lambda$ has all forms of $\Sigma, \Lambda$ has, for $i=1, \cdots, p$, a form $\beta_{i}$ involving only $y_{i}$ and the $u$ 's. Let $\beta_{i}$ be taken so as to be of a minimum rank in $y_{i}$. Let $\beta_{i}$ be of order $r_{i}$ in $y_{i}$ and put $F_{i}=\partial \beta_{i} / \partial y_{i, r_{i} \cdot}$ Similarly, let $\gamma_{i}$; $i=1, \cdots, p$, be a form of $\Lambda$, in $z_{i}$ and the $u$ 's alone, which is of a minimum rank in $z_{i}$. Each $\gamma_{i}$ being of order $s_{i}$ in $z_{i}$, let $K_{i}=\partial \gamma_{i} / \partial z_{i, s_{i}}$.

Then no $F_{i}$ or $K_{i}$ is in $\Lambda$.

To fix our ideas, let us suppose that $y_{1}-z_{1}$ is not in $\Lambda$. Consider any solution of $\Lambda$ for which

$$
\left(y_{1}-z_{1}\right) F_{1} \cdots F_{p} K_{1} \cdots K_{p}
$$

(which is not in $\Lambda$ ) does not vanish.

For such a solution, we have

$$
\lambda_{1}=\frac{\lambda_{2}\left(y_{2}-z_{2}\right)+\cdots+\lambda_{p}\left(y_{p}-z_{p}\right)}{y_{1}-z_{1}} .
$$

From (10) we find, for the $j$ th derivative of $\lambda_{1}$, an expression

$$
\lambda_{1 j}=R_{j}\left(\lambda_{2}, \cdots, \lambda_{p} ; y_{1}, \cdots, y_{p} ; z_{1}, \cdots, z_{p}\right),
$$

in which $R_{j}$ is rational in $\lambda_{2}, \cdots, \lambda_{p}$, the $y$ 's, $z$ 's and the derivatives of the foregoing functions, with coefficients in $F$. The denominator in each $R_{j}$ is a power of $y_{1}-z_{1}$.

If an $R_{j}$ involves a derivative of $y_{i}$ of order higher than $r_{i}$, we can get rid of that derivative by using its expression in the derivatives of $y_{i}$ of order $r_{i}$ or less found from $\beta_{i}=0$. Similarly, we transform each $R_{j}$ so as to be of order not exceeding $s_{i}$ in $z_{i}$.

The new expression of each $R_{j}$, which will involve the $u$ 's, will have a denominator which is a product of powers of $y_{1}-z_{1}, F_{i}, K_{i}, i=1, \cdots, p$.

Then, in (10) and (11), only a finite number of functions $y_{i k}, z_{i k}$ will appear. If we use a sufficiently large number of equations (11), we can, using rigorous principles of elimination, obtain from them an algebraic relation among the functions $\lambda_{i k}, u_{i k}$, with coefficients in $\mathcal{F}$, which holds for any solution of $\Lambda$ which does not cause $y_{1}-z_{1}$, any $F_{i}$, or any $K_{i}$, to vanish. Let

$$
D=0
$$

be such a relation, where $D$ is a form in the $u$ 's and $\lambda$ 's, with coefficients in F. Then

$$
D F_{1} \cdots F_{p} K_{1} \cdots K_{p}\left(y_{1}-z_{1}\right)
$$


holds $\Lambda$, so that $D$ is in $\Lambda$. We have thus proved that $\Lambda$ has a form involving only the $u$ 's and $\lambda$ 's.

Let $\Lambda_{1}, \cdots, \Lambda_{r}$ be a set of irreducible systems such that $\Omega$ holds each of them and that every solution of $\Omega$ is a solution of one of them. We suppose each $\Lambda_{i}$ to contain every form which holds that $\Lambda_{i}$. Let $\Lambda_{1}, \cdots, \Lambda_{s}$ each not contain some of the forms $y_{i}-z_{i}$ and let $\Lambda_{s+1}, \cdots, \Lambda_{r}$ each contain all of the forms $y_{i}-z_{i}$. Let $D_{i}$ be a form in $\Lambda_{i}, i=1, \cdots, s$, involving only.the $u$ 's and $\lambda$ 's.

We wish to show the existence in $\mathcal{F}$ of $p$ functions, $\mu_{1}, \cdots, \mu_{p}$, such that, when each $\lambda_{i}$ is replaced by $\mu_{i}$ in $E=D_{1} \cdots D_{s}$, then $E$ does not vanish identically in the $u$ 's.

Let $E$ be written as a polynomial in the $u$ 's and their derivatives, with forms in the $\lambda$ 's as coefficients. Let $K$ be one of the coefficients in $E$. If we can fix each $\lambda_{i}$ in $F$ so that $K$ does not vanish, our result will be established.

Consider any non-constant function $\zeta$ in $\mathcal{F}$ : Let $P$ be a circle in $\mathscr{A}$ in which $\zeta$ is analytic and assumes no value more than once. Any function analytic in $P$ can be approximated arbitrarily closely, in any area interior to $P$, by a polynomial in $\zeta$, hence by a polynomial in $\zeta$ with rational coefficients. All polynomials in $\zeta$ with rational coefficients are in $\mathcal{F}$.

Thus, if $K$ vanishes for all $\lambda$ 's in $\mathcal{F}, K$ vanishes if the $\lambda$ 's are any functions analytic in $P$. This is certainly impossible. Thus, the required $\mu$ 's exist.

The solutions of $\Omega$, for $\lambda_{j}=\mu_{i}, j=1, \cdots, p$, will be solutions of the systems $\Lambda_{i}$ for $\lambda_{j}=\mu_{j}$. Now the solutions with $\lambda_{j}=\mu_{j}$ of each $\Lambda_{i}, i=1, \cdots, s$, have $u$ 's which cause to vanish the form $G$, obtained from $E$ by putting $\lambda_{j}=\mu_{j}$. The solutions of $\Lambda_{s+1}, \cdots, \Lambda_{r}$, even with $\lambda_{j}=\mu_{j}$, have $y_{i}=z_{i}$, $i=1, \cdots, p$.

We have thus the result stated at the head of this $\$ 21$.

22. From this point on, to the end of our paper, we assume $\Sigma$ irreducible.

Let $A, B, G$ be forms in the $u$ 's and $y$ 's, not in $\Sigma, G$ involving only the $u$ 's, which are such that for any two distinct solutions of $\Sigma$, with the same $u$ 's, for which neither $G$ nor $B$ vanishes, $A / B$ gives two distinct functions of $x$.

We have seen that, when $\mathcal{F}$ does not consist entirely of constants, forms $A, B, G$ exist, that, in fact, one may take $B=1$ and take $A$ free of the $u$ 's. On the other hand, when $\mathcal{F}$ contains only constants, there may be no $A, B, G$. Consider, for instance, the system

$$
\frac{d y_{1}}{d x}=0, \quad \frac{d y_{2}}{d x}=0 .
$$

Any rational combination of $y_{1}$ and $y_{2}$ (and of their derivatives) with con- 
stant coefficients, will have a single value for an infinite number of choices of $y_{1}$ and $y_{2}$.

We introduce a new indeterminate, $w$, and form a system $\Lambda$ by adjoining $B w-A$ to $\Sigma$. Let $\Omega$ be the system of all forms in $w$, the $u$ 's and $y$ 's, which vanish for those solutions of $\Lambda$ for which $B \neq 0$. $^{*}$ We shall prove that $\Omega$ is irreducible.

Let $P$ and $Q$ be forms such that $P Q$ holds $\Omega$. For $s$ appropriate, $B^{*} P$ minus a linear combination of $B w-A$ and its derivatives, is a form $R$ free of $w$. We obtain similarly, from a $B^{\natural} Q$, a form $S$ free of $w$. Then $R S$ vanishes for every solution of $\Sigma$ with $B \neq 0$, since every such solution furnishes a solution of $\Omega$. Hence $B R S$ holds $\Sigma$, so that either $R$ or $S$ is in $\Sigma$. If $R$ is in $\Sigma$, $B^{8} P$ vanishes for all solutions of $\Lambda$. Hence $P$ vanishes for all solutions of $\Lambda$ with $B \neq 0$, so that $P$ is in $\Omega$. Thus $\Omega$ is irreducible.

We notice that those forms of $\Omega$ which are free of $w$ are precisely the forms of $\Sigma$.

We shall prove that $\Omega$ has a form in $w$ and the $u$ 's alone.

Let $\beta_{i}, i=1, \cdots, p$, be a form of $\Sigma$ involving only $y_{i}$ and the $u$ 's, of a minimum rank in $y_{i}$. Let $F_{i}$ have its customary significance.

For any solution of $\Omega$ with $B \neq 0$, we write

$$
w=\frac{A}{B} \text {. }
$$

Representing the $j$ th derivative of $w$ by $w_{j}$, we have

$$
w_{j}=R_{j}\left(u_{1}, \cdots, u_{q} ; y_{1}, \cdots, y_{p}\right),
$$

where $R_{j}$ is rational in the $u$ 's, $y^{\prime}$ 's and their derivatives, the order of the highest derivative of $y_{i}$ in $R_{j}$ not exceeding the maximum of the orders of $\beta_{i}, A$ and $B$ in $y_{i} . \dagger$ The denominator of each $R_{j}$ will be a product of powers of $B, F_{1}, \cdots, F_{p}$. Using a sufficient number of relations (12), we eliminateł the $y$ 's and their derivatives, obtaining a relation in $w$ and the $u$ 's,

$$
K=0,
$$

which holds when $B F_{1} \cdots F_{p}$ does not vanish. As $B F_{1} \cdots F_{p}$ is not in $\Omega$, and as $\Omega$ is irreducible, $K$ must be in $\Omega$. This proves our statement.

23. We take, for $\Omega$, a basic system of forms, analogous to (9),

$$
\alpha, \alpha_{1}, \cdots, \alpha_{p}
$$

\footnotetext{
* Of course, forms in $\Omega$ may also vanish when $B=0$.

$\dagger$ We could depress the order in $y_{i}$ to the order of $\beta_{i}$, but this would complicate what follows.

$\ddagger$ It is clear that this elimination is of a perfectly rigorous nature.
} 
in which $\alpha$ involves only $w$ and the $u$ 's, and in which $\alpha_{1}, \cdots, \alpha_{p}$ introduce in succession $y_{1}, \cdots, y_{p}$.

If $\alpha$ is not irreducible as a polypomial in the $u$ 's, $w$ and their derivatives, with coefficients in $\mathcal{F}$, we can replace it by one of its irreducible factors. We assume, therefore, that $\alpha$ is an irreducible form.

We are going to prove that $\alpha_{1}, \cdots, \alpha_{p}$ are of order zero in $y_{1}, \cdots, y_{p}$, and, indeed, that $\alpha_{i}$ is of the first degree in $y_{i}$. Thus, since $\alpha_{i}$ with $i>1$ must be of lower degree in $y_{j}$ than $\alpha_{j}$ with $j<i$, each equation $\alpha_{i}=0$ will express $y_{i}$ rationally in terms of $w$, the $u$ 's and their derivatives.

The determination of the content of $\Sigma$ will, in this way, be made to depend on the determination of the general solution of $\alpha=0$, which equation will be called a resolvent of $\Sigma$.

It is hardly necessary to call attention to the analogy which the introduction of $w$ creates, between the content of $\Sigma$, and a system of $p$ algebraic functions of $q$ variables.

Suppose that $\alpha_{1}$ is of order higher than zero in $y_{1}$. Consider any regular solution of (13) for which $B G \neq 0$. By the final remark of $\$ 20$, such regular solutions exist. Without changing $w$ or the $u$ 's, in the solution, we can alter the initial conditions for $y_{1}$ slightly, obtaining a second regular solution of (13) with $B G \neq 0$. That is, we can solve $\alpha_{1}=0$ for $y_{1}$ with the modified initial conditions, substitute the resulting $y_{1}$ into $\alpha_{2}$, solve $\alpha_{2}=0$ with the same initial conditions for $y_{2}$ which obtained in the first regular solution, and continue, determining each $y_{i}$. Thus, we would have two distinct solutions of $\Omega$, with the same $u$ 's, with $B G \neq 0$, and with the same $w$.

Hence, $\alpha_{1}$ is of zero order in $y_{1}$. Similarly, every $\alpha_{i}$ is of zero order in $y_{i}$. Furthermore, as $\alpha_{i}$ is of lower rank in $y_{j}$ than $\alpha_{j}$, for $j<i, \alpha_{i}$ is of zero order in $y_{i}$ for $j \leqq i$.

We shall now prove that every $\alpha_{i}$ is linear in $y_{i}$.

We start with $\alpha_{p}$. Suppose that $\alpha_{p}$ is not linear in $y_{p}$.

Let $F_{i}=\partial \alpha_{i} / \partial y_{i}$ and let $H_{i}$ be the coefficient of the highest power of $y_{i}$ in $\alpha_{i}$.

By the familiar process of reduction, we can obtain from $B$ a form $B_{1}$, involving $w$, not in $\Omega$, of lower rank than each $\alpha_{i}$ in $y_{i}$ and of lower rank than $\alpha$ in $w$, such that any regular solution of (13), which causes either of the forms $B$ or $B_{1}$ to vanish, causes the other to vanish.

If we can show that the system

$$
\alpha, \alpha_{1}, \cdots, \alpha_{p-1}
$$


has a regular solution* $w, y_{1}, \cdots, y_{p-1}$ for which $\alpha_{p}$ has two distinct solutions in $y_{p}$ with $F_{p} H_{p} B_{1} G \neq 0$, we shall have forced a contradiction.

If we cannot get two distinct solutions of $\alpha_{p}$ of this type, it must be that for every regular solution of (14) with $H_{p} \neq 0, \alpha_{p}$ has a solution for which $F_{p} B_{1} G$ vanishes. $\dagger$

Dividing $F_{p} B_{1} G$ by $\alpha_{p}$, we obtain a form $\beta$, not in $\Omega$, of zero order in the $y$ 's, and of lower degree than $\alpha_{p}$ in $y_{p}$, such that every common solution of $F_{p} B_{1} G$ and $\alpha_{p}$ is a solution of $\beta$.

Of all forms not in $\Omega$, of zero order in the $y$ 's, which are of lower degree than $\alpha_{p}$ in $y_{p}$, and which, for every regular solution of (14) with $H_{p} \neq 0$, have a solution for $y_{p}$ in common with $\alpha_{p}$, let $\gamma$ have a minimum degree in $y_{p}$. Then $\gamma$ must be at least of the first degree in $y_{p}$, else $H_{p} \gamma$ would vanish for all regular solutions of (13) and would be in $\Omega$.

Let $K$ be the coefficient of the highest power of $y_{p}$ in $\gamma$. Then $K$ is not in $\Omega$. For $m$ appropriate,

$$
K^{m} \alpha_{p}=\delta \gamma+\eta,
$$

with $\delta$ of lower degree than $\alpha_{p}$ in $y_{p}$, and $\eta$ of lower degree than $\gamma$ in $y_{p}$. Every common solution of $\alpha_{p}$ and $\gamma$ makes $\eta$ vanish. Then $\eta$ must be in $\Omega$.

Thus $\delta \gamma$ is in $\Omega$, so that $\delta$, which is not zero, is in $\Omega$. Since $K^{m} \alpha_{p}$ is of higher degree in $y_{p}$ than $\eta$, the coefficient of the highest power of $y_{p}$ in $K^{m} \alpha_{p}-\eta=\delta \gamma$ is not in $\Omega$. Then the coefficient of the highest power of $y_{p}$ in $\delta$ is not in $\Omega$. Thus, reducing $\delta$ with respect to $\alpha_{p-1}, \cdots, \alpha$, by the familiar method, we would obtain from $\delta$ a form in $\Omega$, not zero, of lower degree than every $\alpha_{i}$ in $y_{i}$ and of lower rank than $\alpha$ in $w$.

This contradiction proves that $\alpha_{p}$ is linear in $y_{p}$.

We now consider $\alpha_{p-1}$, assuming that it is not linear in $y_{p-1}$. Since $B_{1}$ is of lower degree than $\alpha_{p}$ in $y_{p}, B_{1}$ is free of $y_{p}$. It must be that, for every regular solution of

$$
\alpha, \alpha_{1}, \cdots, \alpha_{p-2}
$$

with $H_{p-1} \neq 0, \alpha_{p-1}$ has a solution which causes $F_{p-1} H_{p} B_{1} G$ to vanish. The proof continues as for $\alpha_{p}$.

In dealing with $\alpha_{p-2}$, we consider that both $B_{1}$ and $H_{p}$ are free of $y_{p-1}$. The proof continues as above.

Thus every $\alpha_{i}$ is linear in $y_{i}$, and each $y_{i}$ has an expression rational in in $w, u_{1}, \cdots, u_{q}$ and their derivatives, with coefficients in $\mathfrak{F}$.

\footnotetext{
* A solution with $F F_{1} \cdots F_{p-1} H H_{1} \cdots B_{p-1} \neq 0$.

$\dagger$ Because (13) has regular solutions, (14) has regular solutions with $H_{p} \neq 0$.

$\ddagger$ After a multiplication by a power of $\boldsymbol{H}_{\boldsymbol{p}}$.
} 
24. We propose to determine which solutions of (13) other than the regular solutions are solutions of $\Omega$.

We notice first that if

$$
u_{1}, \cdots, u_{q} ; w ; y_{1}, \cdots, y_{p}
$$

is a solution of $\Omega$, then

$$
u_{1}, \cdots, u_{q} ; w
$$

belongs to the general solution of $\alpha$.

For, if a form $K$ in the $u$ 's and $w$ vanishes for every solution in the general solution of $\alpha$, then $K$ vanishes for every regular solution of (13) and so is in $\Omega$.

The question then arises as to which solutions of (13), for which $H_{1} \cdots H_{p}$ vanishes, are solutions of $\Omega$.

This question is settled by the method of $\S \S 17,18$. One sees that for a solution

$$
u_{1}, \cdots, u_{q} ; w ; y_{1}, \cdots, y_{p}
$$

of (13) to be a solution of $\Omega$, it is necessary and sufficient that in every area in which the functions of (16) are analytic, a point a exist such that, for every positive integer $m$, and for every $\epsilon>0$, there is a regular solution of (13) in which the values of the functions and their first $m$ derivatives at a differ from the corresponding values for (16) by quantities less than $\epsilon$ in modulus.

It follows, as in $\S 18$, that if a form with coefficients meromorphic in $\mathfrak{A}$, the coefficients not belonging necessarily to $\mathcal{F}$, vanishes for all regular solutions of (13), the form vanishes for all solutions of $\Omega$.

25. We shall derive a result which is, to some extent, a converse of the result of $\S 23$.

Suppose that we have a differential equation

$$
\alpha\left(u_{1}, \cdots, u_{q} ; w\right)=0,
$$

$\alpha$ being an irreducible form, with coefficients in $\mathscr{F}$.

Let there be given $p$ rational combinations of $u_{1}, \cdots, u_{q}$; $w$ and their derivatives, with coefficients in $\mathcal{F}$,

$$
y_{i}=\frac{P_{i}\left(w ; u_{1}, \cdots, u_{q}\right)}{Q_{i}\left(w ; u_{1}, \cdots, u_{q}\right)} \quad(i=1, \cdots, p),
$$

no $Q_{i}$ vanishing for every solution in the general solution of $\alpha$.

Consider any regular solution of (17) and (18), that is, a set

$$
u_{1}, \cdots, u_{q} ; w ; y_{1}, \cdots, y_{p}
$$


consistent with (17), (18), in which $u_{1}, \cdots, u_{q}$; w is a regular solution of (17), and for which, naturally, no $Q_{i}$ vanishes. It can be shown, as in the preceding sections, that there is, for every $i$, a form in $y_{i}$ and the $u$ 's which vanishes for all regular solutions.

Consider the system $\Sigma$ of all forms in the $y$ 's and $u$ 's which vanish for all regular solutions of (17), (18).

We shall prove that $\Sigma$ is irreducible.

Let $R S$ hold $\Sigma$. If we substitute (18) into $R, R$ becomes a rational combination of $u_{1}, \cdots, u_{q} ; w$ and their derivatives

$$
\frac{T\left(w ; u_{1}, \cdots, u_{q}\right)}{U\left(w ; u_{1}, \cdots, u_{q}\right)},
$$

where $U$ is a product of powers of the $Q_{i}$ 's. Similarly, $S$ becomes a rational combination $V / W$ of the $u$ 's, w etc.

For the $w$ and $u$ 's of any regular solution of (17), (18), $T V$ vanishes. This means that, for every regular solution of $\alpha$,

$$
T V Q_{1} \cdots Q_{p}
$$

vanishes. Then either $T$ vanishes for all regular solutions of $\alpha$, or $V$ does. Hence either $R$ vanishes for all regular solutions of (17), (18), or $S$ does.

Thus $\Sigma$ is irreducible. Its content is an irreducible manifold which is contained in every manifold which contains the regular solutions of (17), (18) with $w$ suppressed.

Consider the system $\Omega$ of all forms in the $u$ 's, $y$ 's and $w$, which vanish for the regular solutions of (17), (18). The above discussion shows also that $\Omega$ is irreducible.

The results of this section hold even if $\mathcal{F}$ consists purely of constants.

\section{INVARIANCE OF THE INTEGER $q$}

26. We propose to show that the number $q$ of arbitrary indeterminates depends only upon the system $\Sigma$ and not on the manner in which the u's are selected.

The assumption that $\Sigma$ is irreducible is essential. But it must be realized, in this connection, that $\$ 19$ develops the idea of arbitrary indeterminate in a rather special way.*

\footnotetext{
* Consider the system of equations $u_{1} y_{1}=u_{2} y_{\varepsilon}=u_{3} y_{2}=0$. These equations imply no relations either among the $u$ 's or among the $y$ 's. Still each $u$ appears in a form with $y$ 's alone, and each $y$ appears with $u$ 's alone.
} 
It will suffice to prove that, given any $q+1$ indeterminates among the $u$ 's and $y$ 's,

$$
z_{1}, \cdots, z_{q+1},
$$

there exists a form of $\Sigma$ which involves only the $z$ 's.

Let us suppose that $\mathfrak{F}$ does not consist purely of constants, and let us consider the regular solutions of (13). For $u_{1}, \cdots, u_{q} ; w$ in such a solution, (13) gives a rational expression for each $z_{i}$. If a $z_{i}$ happens to be a $u$, say $u_{j}$, the expression for $z_{i}$ is simply $u_{j}$. We write

$$
z_{i}=R_{i}\left(w ; u_{1}, \cdots, u_{q}\right) \quad(i=1, \cdots, q+1) .
$$

On differentiating (19) repeatedly, we get expressions for the derivatives of the $z$ 's which are rational in terms of the $u$ 's, $w$ and their derivatives. Making use of the relation $\alpha=0$, we transform these expressions so as not to contain derivatives of $w$ of order higher than $r$, the order of the resolvent in $w$.

None of the expressions thus obtained will have a denominator which vanishes for $u$ 's and $w$ in a regular solution of (13).

Now, if we differentiate the $q+1$ relations (19) often enough, the $z$ 's and their derivatives will become more numerous than the $u$ 's, their derivatives and $w, \cdots, w_{r}$.

It follows that there exists a polynomial in the $z$ 's and their derivatives, with coefficients in $F$, which vanishes for all regular solutions of (13). The form thus obtained belongs to $\Sigma$.

Suppose now that $\mathcal{F}$ contains only constants. Let $\mathcal{F}_{1}$ be the field obtained from $\mathcal{F}$ by the adjunction of $x$. Let $\Sigma_{1}, \cdots, \Sigma_{s}$ be irreducible systems in $\mathcal{F}_{1}$ such that $\Sigma$ holds each of them and that every solution of $\Sigma$ is a solution of one of them.*

Suppose that $\Sigma$ has, in $\mathcal{F}$, two sets of arbitrary indeterminates, $u_{1}, \cdots, u_{q}$ and $z_{1}, \cdots, z_{t}$ with $t \neq q$. We are.going to arrive at the contradiction that both the $u$ 's and the $z$ 's are arbitrary for some $\Sigma_{i}$ in $\digamma_{1}$.

Suppose that this is not so, and that each $\Sigma_{i}$ has either a form in the $u$ 's alone or a form in the $z$ 's alone. Then the product of $s$ such forms, one from each $\Sigma_{i}$, will vanish for every solution of $\Sigma$.

Consider then any form $K$, taken from some $\Sigma_{i}$, which is a polynomial in $x$, the $u$ 's and their derivatives, with coefficients in $\mathcal{F}$. Let $K$ be irreducible as a polynomial in $x$ etc. $\dagger$ Let $K^{\prime}$ be the derivative of $K$. Then the resultant of $K$ and $K^{\prime}$ with respect to $x$, which is not zero, vanishes for any $u$ 's which

* Whether $\Sigma$ can be reducible in $\mathcal{F}_{1}$ is a question.

$\dagger$ Irreducibility may certainly be assumed for the $s$ forms considered above. 
make $K$ vanish. The resultant is a form in the $u$ 's, with coefficients in $F$.

Thus, there is a product of $s$ forms, some in the $u$ 's alone, some in the $z$ 's alone, with coefficients in $\mathcal{F}$, which holds $\Sigma$. This cannot be, as $\Sigma$ is irreducible in $\mathcal{F}$. Thus, there is a $\Sigma_{i}$ for which both the $u$ 's and the $z$ 's are arbitrary. This completes the proof.

\section{INVARIANCE OF ORDER OF RESOLVENT}

27. We propose to show that, $u_{1}, \cdots, u_{q}$ being selected, the order with respect to $w$ of the resolvent is independent of the choice of $w$.

Having taken a definite $w$, and having formed the resolvent in $w, \alpha=0$, let us form a second rational combination of the $u$ 's, $y$ 's and their derivatives,

$$
v=\frac{C}{D},
$$

with $v$ meeting all specifications placed above on $w$. Let the resolvent in $v$ be $\beta=0$.

Since $D$ is not in $\Sigma, D$ is not in the system $\Omega$ based on $w$. Hence, there are regular solutions of (13) for which $D \neq 0$. Taking any such regular solution, let the $y$ 's in it, expressed in terms of $w$ and the $u$ 's, be substituted into the expression (20) for $v$. We find, for $v$, an expression in the $u$ 's, $w$ and their derivatives,

$$
v=R\left(w ; u_{1}, \cdots, u_{q}\right) .
$$

Using the equation $\alpha=0$, if necessary, we may suppose that $R$ involves no derivatives of $w$ of order higher than $r$, the order of $\alpha$ in $w$.

We differentiate (21) $r$ times, and find that, $v_{i}$ being the $i$ th derivative of $v$,

$$
v_{i}=R_{i}\left(w ; u_{1}, \cdots, u_{q}\right)
$$$$
(i=1, \cdots, r) \text {, }
$$

each $R_{i}$ being rational, and involving no derivative of $w$ beyond the $r$ th. From (21), (22) and $\alpha=0$, we can eliminate $w, \cdots, w_{r}$, and obtain an algebraic relation

$$
K\left(u_{1}, \cdots, u_{q} ; v\right)=0,
$$

at most of order $r$ in $v$.

The relation (23) holds for any $v$ given by (20), if the $u$ 's and $y$ 's, for which $D \neq 0$, belong to a regular solution of (13). Now, if we replace $v$ in (23) by its expression (20), (23) becomes a relation in the $u$ 's and $y$ 's,

$$
\frac{L\left(u_{1}, \cdots, u_{q} ; y_{1}, \cdots, y_{p}\right)}{D^{m}}=0,
$$


with $m$ a positive integer. Then $L$ vanishes for all $u$ 's and $y$ 's in a regular solution of (13), for which $D \neq 0$. Thus $D L$ is in $\Omega$, and as $D$ is not in $\Omega$, $L$ is in $\Omega$. Hence $L$ is in $\Sigma$, and (23) holds for any $v$ given by (20), where the $u$ 's and $y$ 's are any solution of $\Sigma$ with $D \neq 0$.

If, then, $\Omega^{\prime}$ is the system associated with $v$ as $\Omega$ is with $w, K$, in (23), is in $\Omega^{\prime}$. This proves that the order of $\beta$ in $v$ does not exceed the order of $\alpha$ in $w$. From considerations of symmetry, it follows that the two orders are equal. This proves our statement.

The degree of the resolvent in the highest derivative of $w$ does depend on the manner of choosing $w$. Consider, for instance, the system, irreducible in the field of all rational functions,

$$
\frac{d}{d x} y_{1}=1, y_{2}=y_{1}^{2} .
$$

As the solution of the system is $y_{1}=x+a, y_{2}=(x+a)^{2}$, we may evidently take $w=y_{1}$. The resolvent becomes $d w / d x=1$. On the other hand, if we take $w=y_{1}+y_{2}$, the resolvent becomes of the second degree in $d w / d x$.

The order of the resolvent depends on the choice of the $u$ 's. For instance

$$
\frac{d y_{1}}{d x}-y_{2}=0
$$

is an irreducible system in the field of rational functions. If we let $u_{1}=y_{2}$, we get a resolvent of the first order. If we let $u_{1}=y_{1}$, we get a resolvent of zero order.

\section{AdJUNCTION OF NEW FUNCTIONS TO $\mathfrak{F}$}

28. Assuming $\mathcal{F}$ not to consist purely of constants, we shall study the circumstances under which $\Sigma$ can become reducible through the adjunction of new functions to $\mathcal{F}$. The adjoined functions are assumed to be meromorphic in $\mathfrak{A}$.

We form a resolvent $\alpha=0$ for $\mathcal{F}$, using a $w$ whose denominator, $B$, is unity.

Suppose that the irreducible factors of $\alpha$, in the enlarged field, $F_{1}$, are $\beta_{1}, \cdots, \beta_{s}$.

Then, by $\S 25$, for each $j$ from 1 to $s$, the system of equations

$$
\beta_{j}=0, \alpha_{1}=0, \cdots, \alpha_{p}=0,
$$

where the $\alpha$ 's are those of (13), defines a system $\Sigma_{j}$ of forms in the $u$ 's and $y$ 's, with coefficients in $\mathfrak{F}_{1}, \Sigma_{j}$ being irreducible in $\mathscr{F}_{1}$.

We shall prove that $\Sigma$ holds every $\Sigma_{i}$, that no $\Sigma_{h}$ holds any $\Sigma_{k}$ with $k \neq h$, 
and that every solution of $\Sigma$ is a solution of some $\Sigma_{j}$. Thus, the systems $\Sigma_{j}$ will furnish the resolution of $\Sigma$ into irreducible systems, in $\mathfrak{F}_{1}$.

Every regular solution of $\alpha$ is a regular solution of some $\beta_{j}$. First, in no $\beta_{j}$ can the coefficient of the highest power of $w_{r}^{*}$ vanish for a regular solution of $\alpha$. Again, since

$$
\frac{\partial \alpha}{\partial w_{r}}=\beta_{2} \cdots \beta_{s} \frac{\partial \beta_{1}}{\partial w_{r}}+\cdots,
$$

$\partial \beta_{j} / \partial w_{r}$ cannot vanish for a regular solution of $\alpha$ if $\beta_{j}$ does. Thus every regular solution of (13) is a regular solution of some system (24). Hence a solution of $\Sigma$ obtained by suppressing $w$ in a regular solution of (13) is a solution of some $\Sigma_{j}$. Since $B=1$, every solution of $\Sigma$ is obtained from some solution of $\Omega$.

Suppose now that some solution of $\Sigma$ is not a solution of any $\Sigma_{j}$. Let $C_{j}$ be a form of $\Sigma_{j}, j=1, \cdots, s$, which does not vanish for the solution. Then $C_{1} \cdots C_{s}$ does not vanish for the solution. This contradicts the final remark of $\$ 24$. Hence every solution of $\Sigma$ is a solution of some $\Sigma_{j}$.

Let $\Omega_{j}(j=1, \cdots, s)$ be the system of all forms in $w$, the $u$ 's and $y$ 's, with coefficients in $\mathcal{F}_{1}$, which vanish for all regular solutions of (24). As was seen in $\$ 25, \Omega_{j}$ is irreducible.

Let $H$ be the coefficient of the highest power of $w_{r}$ in $\alpha$. If $H$ were in some $\Omega_{j}$, it would vanish for all regular solutions of $\beta_{j}$. This cannot be, for $H$ is of order less than $r$ in $w$.

If $F=\partial \alpha / \partial w_{r}$ were of order $r$ and were in some $\Omega_{j}$, it would be divisible by $\beta_{j}$. Then $\alpha$ would be reducible in $\mathfrak{F}$.

Consider any form $P$ of $\Omega$. Any regular solution of (24), for any $j$, for which $F H$ does not vanish, causes $P$ to vanish. Hence $F H P$ is in $\Omega_{j}$, so that $P$ is in $\Omega_{j}$. Thus every form of $\Sigma$ is in $\Sigma_{j}$, so that $\Sigma$ holds every $\Sigma_{j}$.

The foregoing shows also that $\Omega$ holds every $\Omega_{j}$. Thus every $\Omega_{j}$ contains the form $w-A$ used in building $\Omega$. It follows easily that $\Omega_{j}$ holds and is held by the system $\Lambda_{j}$ obtained by adjoining $w-A$ to $\Sigma_{j}$.

This means that if $\Sigma_{h}$ held $\Sigma_{k}$, where $k \neq h$, then $\Omega_{h}$ would hold $\Omega_{k}$. Then $\beta_{h}$ would be in $\Omega_{k}$, and would be divisible by $\beta_{k}$. This would make $\alpha$ reducible in $\mathcal{F}$. Thus no $\Sigma_{h}$ can hold a $\Sigma_{k}$ with $k \neq h$.

Thus, for $\Sigma$ to be reducible in $\mathfrak{F}_{1}$, it is necessary and sufficient that the resolvent of $\Sigma$ relative to $\mathcal{F}$ be algebraically reducible in $\mathcal{F}_{1} . \dagger$

29. The question might be asked as to whether $\Sigma$, irreducible in $\mathcal{F}$ for

* $\alpha$ of order $r$ in $w$.

$\dagger$ We recall the assumption that $B=1$. 
the area $\mathfrak{A}$, can be reducible in $\mathcal{F}$ for some area $\mathfrak{B}$ contained in $\mathfrak{A}$. We shall show that the answer is negative.

We begin by showing that if a form $K$, with coefficients in $\digamma$, vanishes for all solutions of $\Sigma$ analytic in a part of $\mathscr{B}$, then $K$ vanishes for all solutions of $\Sigma$. Suppose then that $K$ is not in $\Sigma$. By the familiar process of reduction, we obtain from $K$ a form $L$, in $w$ and the $u$ 's, of lower rank in $w$ than $\alpha$, which vanishes for every regular solution of $\alpha$, analytic in a part of $\mathfrak{B}$, for which no $H_{i}$ vanishes.* As in $\S 15$, we reach the absurdity that

$$
\mathrm{LFHH}_{1} \cdots \mathrm{H}_{p}
$$

is divisible by $\alpha . \dagger$

Now if $P$ and $Q$ are two forms, with coefficients in $F$, such that $P Q$ vanishes for all solutions of $\Sigma$ analytic in a part of $\mathscr{B}$, than $P Q$ vanishes for all solutions analytic in any part of $\mathscr{A}$. This means that either $P$ or $Q$ is in $\Sigma$, so that $\Sigma$ is irreducible in $\mathfrak{B}$.

* $H_{i}$ is the coefficient of $y_{i}$ in $\alpha_{i}$.

$\dagger$ When $\mathcal{F}$ contains only constants, we adjoin $x$ to $\mathcal{F}$ and consider the irreducible systems into which $\mathbf{\Sigma}$ decomposes.

Columaia University, NEW YoRK, N. Y. 